\title{
Enhancement of quality of service (QoS) in IEEE 802.11
}

\author{
Ababakr lbrahim Rasul \\ Department of Computer Science, Faculty of Science, Soran University, Kurdistan Region - Iraq \\ Ababakr.rasul@soran.edu.iq \\ Diyar Salah Fadhil \\ Department of Computer Science, Faculty of Science, Soran University, Kurdistan Region - Iraq \\ Diyar.fadhil@soran.edu.iq
}

\begin{abstract}
Today everyone is living in a gold rush of wireless communication worldwide. If we look around, we will find wireless communication happening everywhere. In our home, small routers are providing us with wireless access to the internet in offices, shopping centers, cafes, universities, and so on. In this article, a mechanism has been introduced by combining service differentiation and packet aggregation in IEEE 802.11-based WMNs. The result is IEEE802.11b is the most reliable standard among all other wireless standards. Changes required in modulation techniques so that their overhead can be decreased and data can travel in more effective and efficient way.
\end{abstract}

\section{Keyword}

IEEE 802.11, RTS/CTS, DCF, quality of service (QoS)

\section{INTRODUCTION}

$\mathrm{Wi}-\mathrm{Fi}$ hotspots are available all around, most of these Wireless local area networks relay on IEEE 802.11 standards (Stallings, 2009).

According to Akyildiz et al (2005), wireless Mesh Networks (WMNs) is becoming popular in the last few years. These networks are now commonly used for broadband ubiquitous Internet access.

In this article, a mechanism has been introduced by combining service differentiation and packet aggregation in IEEE 802.11-based WMNs. This architecture does not require any modification to the IEEE 802.11 MAC and can be readily deployed exploiting off-the-shelf hardware. The proposed solution has been implemented as an extension to the MIT Roofnet platform. Measurements run over a WiFi testbed show a large gain in the voice capacity attained (Powell and Shim , 2009)

WMNs exploit a multi-hop wireless back-haul in order to deliver Internet connectivity to end-users. Even though WMNs could be interface, through suitable gateways, networks based on different radio technologies (3G, WiMAX,WiFi, Bluetooth), and in this paper IEEE 802.11-based WMNs has been discussed.

The demand of applications running on wireless technology has increased since the use and demand of wireless networking has increased (Chilamkurti et al , 2013). The use of multimedia applications like audio and video became very high. In order to run all these applications, some sort of standardization and support are necessary. The IEEE has taken action by setting up committees to establish standards for wireless LAN. In 1997, the Institute of Electrical and Electronics Engineers (IEEE) formed the earliest WLAN standard. They named it 802.11. The first version of this standard was released in mid-1999. The frequency of 802.11 was $2.4 \mathrm{GHz}$ and data rate was $1-2 \mathrm{Mbps}$. However, at the end of the same year, 802.11b specification was released (Philip. 2005).

Since wireless LAN technology was introduced in public, its use and demand increased in the marked at a very high pace. The main reasons for its popularity include the no necessity for heavy cabling installation, cheap cost and time saving. IEEE introduced the first version of WLAN 802.11 in 1997 and after two years introduced two more versions "a" and "b". 802.11b was adopted in the market very quickly all around the world. A lot of new versions have been introduced but 802.11b is still widely used (Pahlavan and Levesque, 2005). In last 10 years, the use of computer devices like laptops, iPads and smart phones, to browse the internet has increased. All these devices are coming with the capability to connect with local Wi-Fi hot spots or any WLANs. Every day, new gadgets and different types of applications are being introduced in the market. The use of internet, multimedia, audio and video is increasing very quickly. Now WLANs have to handle heavy load and data traffic. The performance of wireless networks generally depends on end to end delay, throughput and bandwidth utilization. Different applications have different necessities on a network. DCF is the main medium access control (MAC) method in $802.11 \mathrm{~b}$, and it is important for the analysis of all quality of service (QoS) parameters on IEEE $802.11 \mathrm{~b}$ to assess its performance ( Liu et al , 2010).

The 802.11 architecture is built-up with different elements and services that act together to present mobile stations and transparent to upper layers of the network batch. In this research, the authors aim at enhancing the perceived quality of experience combining service differentiation and packet aggregation in IEEE 802.11-based WMNs.

\section{RESEARCH LITERATURE}

A study by Vardakas et al (2007) conducted an End-to-End delay analysis of the performance of IEEE 802.11b MAC protocol to resolve the contention problem between WLAN stations and for the authentication of successful transmission. This study relayed on Bianchi's model for DCF analysis. For the probability distribution to obtain a mean value and a variance of MAC delay, a z-transform of backoff period is used. After obtaining the value of the mean and the variance for the MAC delay, they conclude the mean waiting delay for each station. Their analysis is confirmed by simulation outputs of 
DCF for both the basic access system and the RTS/CTS access method. The correctness of the analysis was found quite acceptable. The data rate was supposed 1,5.5 and $11 \mathrm{Mbps}$ sequentially to show up the result of the bit rate on the performance of delay in both access methods. The analysis is also confirmed by simulation outcome using the NS-2 simulator. The simulation was produced for both access methods of IEEE 802.11b DCF the basic access method and RTS/CTS access method (Perahia, and Stacey, 2008).

Another study of 802.11 WLAN by using distributed coordination function is presented in this paper (Naru, et al, 2009). In this research, opposed stations were assumed in a saturated atmosphere within the coverage of an AP, in analysis of unstable data rates and container sizes in a fault free environment. It is been to calculate the delay, and through the performance of Distributed Coordinated Function of IEEE $802.11 \mathrm{~b}$ simulation model is used. This simulation model is supposed to have a fixed number of saturated position (nodes) broadcasting packets on a perfect channel and employ Basic Access Method for obtaining a channel for communication in a solo BSS (Szczypiorski and Lubacz, 2008). This model is confirmed by evaluating its outcome with other simulated and analytical representations. The calculated results prove that the performance of the basic access technique used for IEEE 802.11 primarily depends on the quantity of stations in the wireless network. It is also calculated that by using reduced packet size, the contention in the channel among the stations turn out to be higher sourcing high delay and if these packets are transmitted ahead at low data rate in the result, the band width overhead will decrease in the throughput. Nevertheless, by having large or small packet size and high data rate with large number of nodes, produces in additional channel consumption improves on the whole network performance and basis increase in throughput and reduces delay.

\section{RESEARCH METHODS, TOOLS AND TECHNIQUES}

The research method used in this article is qualitative, as it focused on a traffic aggregation and differentiation scheme for enhanced QoS in IEEE 802.11-based Wireless Mesh Networks.

The tested scheme over an IEEE 802.11-based WMN is deployed exploiting both VolP flows and saturated TCP connections.

In order to validate the adaptive packet aggregation policy, importance was given to VoIP applications. In order to highlight the impact of aggregation scheme, the test performed during the measurement campaign refers to downlink traffic only.

\section{OUTCOME AND CONCLUSION:}

Many authors have contributed in the study of IEEE standards. They used different tools and formulas to analyze the functions and performance of 802.11 according to different QoS parameters.

To Sum up, this traffic aggregation and differentiation scheme is best if we need secure communication, which is achieved through DCF and RTS/CTS mechanisms in wireless mesh networks. IEEE802.11b is the standard which is most reliable in all wireless standards, so changes required in modulation techniques in order to decrease its overhead so data can travel in more effective and efficient way. Moreover, according to network capability, fragmentation factor should be adjusted, which will not only increase network throughput but also will achieve better quality of service.

\section{REFERENCES:}

i. $\quad$ Akyildizi, w x, Wang, W, 2005, Wireless mesh networks: a survey,Elsevier Computer Networks, 47 (4), p 445487.

ii. Chilamkurti ,N, Zeadally ,S and Chaouchi, H ,2013, Next-Generation Wireless Technologies: 4G and Beyond,USA, springer

iii. Liu ,R, Sutton, G and Collings, I, 2010, A New Queueing Model for QoS Analysis of IEEE 802.11 DCF with Finite Buffer and Load, IEEE Communications Society,9 , (8), pp. 2664-2675,

iv. Naru,M, Nauman, $U$ and Hussain,K 2009, Performance Analysis of Wireless Local Area Networks, International Journal of Computer Theory and Engineering,1 (2), p 140 - 145.

v. Pahlavan, K and Levesque , A, 2005, Wireless Information Networks, $2^{\text {nd }}$ ed,US, John Wiley

vi. Perahia, E and Stacey,R, 2008, Robert Next Generation Wireless LANs: Throughput, Robustness, and Reliability in $802.11 \mathrm{n}$, UK, Cambridge University Press.

vii. PHILIP, (2005) Wireless LAN Standard, Retrieved from http://www.speedguide.net/articles/wireless-lanstandards-1582.

viii. Powell , T and Shim ,J , 2009, Wireless Technology: Applications, Management, and Security, USA, springer science

ix. Stallings , W, ,2009, Wireless Communications \& Networks ,USA ,Publisher Pearson Education,

x. Szczypiorski, K and Lubacz,, J ,2008, Saturation throughput analysis of IEEE 802.11g (ERP-OFDM) networks, Telecommunications Systems, 38 2), p.45-52

xi. Vardakas, J, Papapanagiotou, M, Logotheti, S and Kotsopoulos ,S 2007 On the End-to-End Delay Analysis of the IEEE 802.11 Distributed Coordination Function, Proceeding of Second International Conference on Internet Monitoring and Protection (ICIMP) 